\title{
PROPRIÉTÉS DES SOLUTIONS DU SYSTÈME PARABOLIQUE D'ÉQUATIONS AUX DÉRIVÉES PARTIELLES
}

W. POGORZELSKI

1. Introduction et les formules fondamentales. Soit le système de $N \geqq 1$ équations aux dérivées partielles d'ordre $M \geqq 2$ de la forme

$$
\begin{aligned}
& \hat{\Psi}^{(\alpha)}\left(u_{1}, \ldots u_{N}\right)=\sum_{1 \leqq j \leqq N}^{k_{1}+\ldots+k_{n}=M} A_{\alpha j}^{k_{1} \ldots k_{n}}(X, t) \frac{\partial \boldsymbol{M}_{j}}{\partial x_{1}^{k_{1}} \ldots \partial x_{n}{ }^{k_{n}}}+ \\
& +\sum_{1 \leqq j \leqq N}^{1 \leqq k_{1}+\ldots+k_{n}<M} B_{\alpha j}^{k_{1} \ldots k_{n}}(X, t) \frac{\partial^{k_{1}+\ldots+k_{n}} u_{j}}{\partial x_{1}^{k_{1}} \ldots \partial x_{n}{ }^{k_{n}}}+ \\
& +\sum_{1 \leqq j \leqq N} C_{\alpha j}(X, t) u_{j}-\frac{\partial u_{\alpha}}{\partial t}=0
\end{aligned}
$$

$(\alpha=1,2, \ldots, N)$, où $u_{1}(X, t), \ldots, u_{N}(X, t)$ sont des fonctions inconnues dans la région

$$
X \in \Omega ; \quad 0 \leqq t \leqq T
$$

$\Omega$ désigne un domaine borné mesurable et fermé dans l'espace euclidien $E$ des points $X\left(x_{1}, x_{2}, \ldots, x_{n}\right)$.

Nous admettons pour les coefficients des équations (1) les hypothèses suivantes, les mêmes que dans notre travail [5] et beaucoup plus générales que dans le travail [1].

I. Les coefficients $A_{\alpha j}(X, t)$ des dérivées d'ordre le plus élévé $M$ sont des fonctions complexes, déterminées dans la région fermée (2) et vérifiant la condition de Hölder par rapport aux variables spatiales et la variable $t$ de la forme

$$
\left|A_{\alpha j}^{k_{1} \ldots k_{n}}(X, t)-A_{\alpha j}^{k_{1} \ldots k_{n}}\left(X_{1}, t_{1}\right)\right|<\text { const. }\left[\left|X X_{1}\right|^{h}+\left|t-t_{1}\right|^{h^{\prime}}\right]
$$

$(\alpha, j=1,2, \ldots N)$, où $h$ et $h^{\prime}$ sont des constantes positives, non supérieures à l'unité, $\left|X X_{1}\right|$ désigne la distance euclidienne des deux points arbitraires $X$ et $X_{1}$ de l'espace $E, t$ et $t_{1}$ sont les deux valeurs arbitraires de l'intervalle $(0, T)$.

Reçu le 25 avril, 1958. 
II. Les coefficients $B_{\alpha j}, C_{\alpha j}$ des dérivées d'ordre inférieur à $M$ sont des fonctions complexes, déterminées dans la région fermée (2), vérifiant la condition de Hölder par rapport aux variables spatiales

$$
\left\{\begin{array}{l}
\left|B_{\alpha j}^{k_{1} \ldots k_{n}}(X, t)-B_{\alpha j}^{k_{1} \ldots k_{n}}\left(X_{1}, t\right)\right|<\text { const. }\left|X X_{1}\right|^{h} \\
\left|C_{\alpha j}(X, t)-C_{\alpha j}\left(X_{1}, t\right)\right|<\text { const. }\left|X X_{1}\right|^{h}
\end{array}\right.
$$

$(\alpha, j=1,2, \ldots N)$ et sont continues par rapport à la variable $t$. La condition de Hölder par rapport à cette variable n'est pas nécessaire.

III. Conformément à la définition du système parabolique, donnée par Petrovsky [3], l'équation

$$
\operatorname{det}\left|\sum_{\alpha, j} A_{k_{1}+\ldots+k_{n}=M} A_{\alpha j}^{k_{1} \ldots k_{n}}(X, t)\left(i s_{1}\right)^{k_{1}} \ldots\left(i s_{n}\right)^{k_{n}}-\lambda \delta_{\alpha}^{j}\right|=0
$$

admet les racines en $\lambda$, dont toutes les parties réelles sont inférieures où égales à un nombre négatif fixé $-\delta$

$$
\operatorname{Re}(\lambda) \leqq-\delta<0
$$

pour toutes les valeurs des variables réelles $s_{1}, s_{2}, \ldots, s_{n}$ vérifiant l'égalité

$$
s_{1}^{2}+s_{2}^{2}+\ldots+s_{n}^{2}=1
$$

et pour tout point $(X, t)$ de la région $(2) ; \delta_{\alpha}^{j}$ désigne le symbole de Kronecker. Cette hypothèse exige donc d'admettre que le degré $M$ des équations (2) est pair.

En vue des considérations ultérieures nous prolongeons arbitrairement les coefficients $A_{\alpha j}, B_{\alpha j}, C_{\alpha j}$ en tout l'espace $E$, mais sous la condition que les hypothèses I, II, III soient satisfaites et que ces coefficients soient bornés en tout l'espace $E$ pour $0 \leqq t \leqq T$.

La construction de la matrice des solutions fondamentales du système (1), que nous avons donné dans le travail [5], exige de la détermination d'abord de la matrice des quasi-solutions. Les éléments de cette matrice sont donnés par les intégrales de Fourier

$$
W_{\alpha \beta}^{Z, \zeta}(X, t ; Y, \tau)=\frac{1}{(2 \pi)^{n}} \int_{-\infty}^{+\infty} v_{\alpha}^{\beta}(t, \tau ; Z, \zeta ; S) \exp \left\{i \sum_{\nu=1}^{n} s_{\nu}\left(x_{v}-\xi_{v}\right)\right\} d s_{1} \ldots d s_{n}
$$

$(\alpha, \beta=1,2, \ldots, N)$ étendues à tout l'espace euclidien $E$ des variables réelles $S\left(s_{1}, s_{2}, \ldots, s_{n}\right)$. Les symboles $X\left(x_{1}, \ldots, x_{n}\right)$ et $Y\left(\xi_{1}, \ldots, \xi_{n}\right)$ désignent deux points arbitraires de l'espace $E ; Z$ désigne un point arbitrairement fixé dans l'espace $E$, et $\zeta$ une valeur arbitrairement fixée à l'intérieur de l'intervalle $(0, T)$. Les fonctions 


$$
v_{1}^{\beta}, v_{2}^{\beta}, \ldots, v_{N}^{\beta} \quad(\beta=1,2, \ldots, N)
$$

pour $\beta$ fixé forment une solution du système d'équations différentielles ordinaires

$$
\frac{d v_{\alpha}^{\beta}}{d t}=\sum_{1 \leqq j \leqq N}^{k_{1}+\ldots+k_{n}=M} A_{\alpha j}^{k_{1} \ldots k_{n}}(Z, \zeta)\left(i s_{1}\right)^{k_{1}} \ldots\left(i s_{n}\right)^{k_{n}} v_{j}^{\beta}(t, \tau ; Z, \zeta ; S)
$$

où $Z, \zeta, S, \tau$ jouent un rôle des paramètres fixés; on suppose

$$
0 \leqq \tau<t \leqq T .
$$

On demande, en outre, que les solutions du système (10) vérifient la condition initiale

$$
\lim _{t \rightarrow \tau} v_{\alpha}^{\beta}(t, \tau ; Z, \zeta ; S)=\delta_{\alpha}^{\beta} \quad \text { (symbole de Kronecker) }
$$

On démontre (voir [5]) que les fonctions $v_{\alpha}^{\beta}$ (entières par rapport aux variables $\left(s_{1}, \ldots, s_{n}\right)$ vérifient l'inégalité importante

$$
\left|v_{\alpha}^{\beta}(t, \tau ; Z, \zeta ; S)\right|<K e^{-\frac{1}{2} \delta s^{M}(t-\tau)}
$$

où

$$
s=\left(s_{1}^{2}+\ldots+s_{n}^{2}\right)^{\frac{1}{2}} \quad\left(s_{\nu} \text { étant réelles }\right) .
$$

$\delta$ est la constante positive, fixée par l'hypothèse (6). $K$ est une constante positive, indépendante des paramètres $Z, \zeta, S, \tau$. L'inégalité (13) assure la convergence absolue des intégrales (8) et nous permet de conclure que les éléments de la matrice (8) possèdent les dérivées des tous les ordres par rapport aux coordonnées du point $X\left(x_{1}, \ldots, x_{n}\right)$, données par les formules

$$
=\frac{1}{(2 \pi)^{n}} \int_{-\infty}^{+\infty} v_{\alpha}^{\beta}(t, \tau ; Z, \zeta ; S)\left(i s_{1}\right)^{k_{1}} \ldots\left(i s_{n}\right)^{k_{n}} \exp \left\{i \sum_{\nu=1}^{n} s_{v}\left(x_{\nu}-\xi_{v}\right)\right\} d s_{1} \ldots d s_{n} .
$$

Les intégrales (14) sont absolument convergentes pour toutes les valeurs non-négatives des entières $k_{1}, k_{2}, \ldots, k_{n}$. Remarquons, en outre, que les fonctions $v_{\alpha}^{\beta}$, d'après les équations (10), admettent les dérivées par rapport à la variable $t$, qui aussi vérifient l'inégalité de la forme (13), donc les intégrales (8) sont aussi dérivables par rapport à la variable $t$.

En s'appuyant sur les égalités (14) et sur le système (10), nous en concluons que les $N$ suites $(\beta=1,2, \ldots, N)$ de fonctions

$$
W_{1 \beta}^{Z, \zeta}, W_{2 \beta}^{Z, \zeta}, \ldots, W_{N \beta}^{Z, \zeta}
$$


formes par les éléments de la matrice $\left\|W_{\alpha \beta}^{Z, \zeta}\right\|$, présentent $N$ solutions du système d'équations suivantes

$$
\sum_{1 \leqq j \leqq N}^{k_{1}+\ldots+k_{n}=M} A_{\alpha j}^{k_{1} \ldots k_{n}}(Z, \zeta) \frac{\partial^{M} W_{j \beta}^{Z, \zeta}}{\partial x_{1}^{k_{1}} \ldots \partial x_{n}{ }^{k_{n}}}-\frac{\partial W_{\alpha \beta}^{Z, \zeta}}{\partial t}=0,
$$

où les coefficients sont fixés au point $(Z, \zeta)$ arbitrairement choisi. C'est pourquoi nous avons appelé les suites (15) - quasi-solutions du système (1) et la matrice des éléments (8) - matrice des quasi solutions du système (1).

On peut montrer (voir les travaux [5] et [1]), en s'appuyant sur un théorème de Gelfand-Chilov [2], que les éléments de la matrice des quasisolutions vérifient les inégalités

$$
\left|W_{\alpha \beta}^{Z, \zeta}(X, t ; Y, \tau)\right|<\frac{C}{(t-\tau)^{n / M}} \exp \left[-\frac{c|X Y|^{q}}{(t-\tau)^{1 /(M-1)}}\right],
$$

où $q=M /(M-1) ; C, c$ sont des constantes positives indépendantes de $Z, \zeta$; $X, Y$ sont deux points arbitraires de l'espace $E$, et $0 \leqq \tau<t \leqq T$.

De l'inégalité (17) résultant les limitations suivantes aux singularités séparées (voir [5]), si $X \neq Y, t<\tau$,

$$
\begin{aligned}
& \left|W_{\alpha \beta}^{Z, \zeta}(X, t ; Y, \tau)\right|<\frac{\text { const. }}{(t-\tau)^{\mu}} \frac{1}{|X Y|^{n-M \mu}}, \\
& \left|W_{\alpha \beta}^{Z, \zeta}(X, t ; Y, \tau)\right|<\frac{\text { const. }(t-\tau)^{\mu^{\prime}}}{|X Y|^{n+M \mu^{\prime}}},
\end{aligned}
$$

où $\mu$ est une constante arbitrairement choisie à l'intérieur de l'intervalle $(0,1)$, si $n \geqq M$ et $0<\mu<n M^{-1}$, si $n<M, \mu^{\prime}$ - une constante positive arbitraire. D'une façon analogue, d'après la formule (14), nous avons obtenue la limitation suivante pour les dérivées spatiales d'ordre $m$ quelconque des éléments de la matrice (8)

$$
\left|D_{X}^{(m)}\left[W_{\alpha \beta}^{Z, \zeta}(X, t ; Y, \tau)\right]\right|<\frac{C_{m}}{(t-\tau)^{(n+M) / M}} \exp \left[-\frac{c_{m}|X Y|^{q}}{(t-\tau)^{1 /(M-m)}}\right] .
$$

$C_{m}$ et $c_{m}$ étant des constantes positives, indépendantes de $Z, \zeta$. Il en résultent les limitations aux singularités séparées

$$
\begin{aligned}
\left|D_{X}^{(m)}\left[W_{\alpha \beta}^{Z, \zeta}(X, t ; Y, \tau)\right]\right| & <\frac{\text { const. }}{(t-\tau)^{\mu}} \frac{1}{|X Y|^{n+m-M \mu}}, \\
\left|D_{X}{ }^{(m)}\left[W^{Z, \zeta}(X, t ; Y, \tau)\right]\right| & <\frac{\text { const. }(t-\tau)^{\mu^{\prime}}}{|X Y|^{n+m+M \mu^{\prime}}},
\end{aligned}
$$


où $0<\mu<1$, si $n+m \geqq M$ et $0<\mu<(n+m) M$, si $n+m<M, \mu^{\prime}>0, X \neq Y$. Nous ferons l'usage dans les considérations ultérieures des inégalités (18) et (20), si $|X Y| \rightarrow 0$, et des inégalités $\left(18^{\prime}\right)$ et $\left(20^{\prime}\right)$, si $|X Y| \rightarrow \infty$.

D'après le travail [5], les éléments $\Gamma_{\alpha \beta}(X, t ; Y, \tau)$ de la matrice des solutions fondamentales sont donnés par la formule ${ }^{1}$

$$
\begin{aligned}
\Gamma_{\alpha \beta}(X, t ; Y, \tau)= & W_{\alpha \beta}^{Y, \tau}(X, t ; Y, \tau)+ \\
& +\int_{\tau}^{t} \iiint_{E}^{t} \int_{(I)} \sum_{\gamma=1}^{N} W_{\alpha \gamma}^{\Pi, \zeta}(X, t ; \Pi, \zeta) \Phi_{\gamma \beta}(\Pi, \zeta ; Y, \tau) d \Pi d \zeta,
\end{aligned}
$$

où $X\left(x_{1}, \ldots, x_{n}\right)$ et $Y\left(\xi_{1}, \ldots, \xi_{n}\right)$ sont les deux points différents, arbitraires, de l'espace $E$ et $\tau<t$ - deux valeurs arbitraires dans l'intervalle $(0, T)$. Les fonctions $\Phi_{1 \beta}, \Phi_{2 \beta}, \ldots, \Phi_{N \beta}$ des couples $\Pi, \zeta$ et $Y, \tau$ (où $\tau<\zeta$ ) forment pour chaque $\beta$ fixé une solution du système d'équations intégrales de Volterra

$$
\begin{aligned}
& \Phi_{\alpha \beta}(X, t ; Y, \tau)=\hat{\Psi}_{(j) X, t}^{(\alpha)}\left[W_{j \beta}^{Y, \tau}(X, t ; Y, \tau)\right]+ \\
& \quad+\int_{\tau}^{t} \iiint_{E} \int_{(I)} \sum_{\gamma=1}^{N} \hat{\Psi}_{(j) X, t}^{(\alpha)}\left[W_{j \gamma}^{\Pi, \zeta}(X, t ; \Pi, \zeta)\right] \Phi_{\gamma \beta}(\Pi, \zeta ; Y, \tau) d \Pi d \zeta
\end{aligned}
$$

$(\alpha=1,2, \ldots, N)$, où $\hat{\Psi}_{(j) X, t}^{(\alpha)}\left(u_{j}\right)$ désigne une opération différentielle sur le système de fonctions $u_{j}\left(u_{1}, u_{2}, \ldots, u_{N}\right)$ par rapport aux variables $X, t$, définie par les formules $(1) ; Y, \tau$ jouent un rôle des paramètres fixés.

La formule (21) fait connaître les $N$ systèmes de fonctions

$$
\Gamma_{1 \beta}(X, t ; Y, \tau) ; \ldots ; \Gamma_{N \beta}(X, t ; Y, \tau)
$$

qu'on obtient en substituant $\beta=1,2, \ldots, N$ et qui vérifient le système d'équations données (1)

$$
\hat{\Psi}_{X, t}^{(\alpha)}\left[\Gamma_{1 \beta}(X, t ; Y, \tau) ; \ldots ; \Gamma_{N \beta}(X, t ; Y, \tau)\right]=0
$$

$(\alpha=1,2, \ldots, N)$ si $X \neq Y ; 0<\tau<t<T$.

Nous avons démontré dans le travail [5], que les solutions du système d'équations intégrales (22) vérifient les inégalités

$$
\left|\Phi_{\alpha \beta}(X, t ; Y, \tau)\right|<\frac{\text { const. }}{(t-\tau)^{\mu_{1}}} \cdot \frac{1}{|X Y|^{n+M\left(1-\mu_{1}\right)-h_{1}}},
$$

si $|X Y| \leqq R_{0}, 1-h_{1} M^{-1}<\mu_{1}<1$ et les inégalités

1 Nous désignons dans ce travail par le symbole d'intégrale triple - l'intégrale de volume dans l'espace à $n$ dimensions, $d \Pi$ désigne l'élément de volume au point $\Pi$. 


$$
\left|\Phi_{\alpha \beta}(X, t ; Y, \tau)\right|<\frac{\text { const. }(t-\tau)^{\mu_{1}^{\prime}}}{|X Y|^{n+M \mu_{1}^{\prime}}},
$$

si $|X Y|>R_{0}\left(\mu_{1}^{\prime}>0\right)$, où $h_{1}=\min \left(h, 2 h^{\prime}\right)$ (voir les inégalités (3)), $R_{0}$ est un nombre positif fixé arbitrairement.

2. Limitations des solutions fondamentales. Écrivons, d'après la formule (21),

$$
\Gamma_{\alpha \beta}(X, t ; Y, \tau)=W_{\alpha \beta}^{Y, \tau}(X, t ; Y, \tau)+\bar{W}_{\alpha \beta}(X, t ; Y, \tau),
$$

en posant

(26) $\bar{W}_{\alpha \beta}(X, t ; Y, \tau)=\int_{\tau}^{t} \iiint_{E} \int_{(I)} \sum_{\gamma=1}^{N} W_{\alpha \gamma}^{\Pi, \zeta}(X, t ; \Pi, \zeta) \Phi_{\gamma \beta}(\Pi, \zeta ; Y, \tau) d \Pi d \zeta$.

En tenant compte des inégalités $(18),\left(18^{\prime}\right),(24),\left(24^{\prime}\right)$, nous en déduirons (voir [5]) pour la fonction (26) la limitation suivante

$$
\left|\bar{W}_{\alpha \beta}(X, t ; Y, \tau)\right|<\frac{\text { const. }}{(t-\tau)^{\mu+\mu_{1}-1}} \cdot \frac{1}{|X Y|^{n-M\left(\mu+\mu_{1}-1\right)-h_{1}}},
$$

si $|X Y| \leqq R_{0}$, et

$$
\left|\bar{W}_{\alpha \beta}(X, t ; Y, \tau)\right|<\frac{\text { const. }\left.(t-\tau)\right|^{\mu^{\prime}}}{|X Y|^{n+M \mu^{\prime}}},
$$

si $|X Y|>R_{0}$. Nous voyons que les singularités de la limitation (27) sont plus faibles que celles de la limitation (18), nous aurons donc

$$
\left|\Gamma_{\alpha \beta}(X, t ; Y, \tau)\right|<\frac{\text { const. }}{(t-\tau)^{\mu}} \cdot \frac{1}{|X Y|^{n-M \mu}} \quad(X \neq Y),
$$

si $|X Y| \leqq R_{0}$ et

$$
\left|\Gamma_{\alpha \beta}(X, t ; Y, \tau)\right|<\frac{\text { const. }(t-\tau)^{\mu^{\prime}}}{|X Y|^{n+M \mu^{\prime}}},
$$

si $|X Y|>R_{0}$, où $0<\mu<1$, dans le cas $n \geqq M$ et $0<\mu<n M^{-1}$, dans le cas $n<M, \mu^{\prime}$ est une constante positive quelconque.

$\mathrm{D}^{\prime}$ après nos études [5], la fonction (26) admet les dérivées d'ordre $m \leqq M-1$ par rapport aux coordonnées du point $X$, données par les formules

$$
\begin{aligned}
D_{X}{ }^{(m)}\left[\bar{W}_{\alpha \beta}(X, t ; Y, \tau)\right] & \\
& =\int_{\tau}^{t} \iiint_{E} \sum_{\gamma=1}^{N} D_{X}{ }^{(m)}\left[W_{\alpha \gamma}^{\Pi, \zeta}(X, t ; \Pi, \zeta)\right] \Phi_{\alpha \beta}(\Pi, \zeta ; Y, \tau) d \Pi d \zeta
\end{aligned}
$$


en tout point $X \neq Y$, pour $0<\tau<t<T$; les intégrales (29) sont absolument convergentes, d'après les limitations $(20),\left(20^{\prime}\right),(24),\left(24^{\prime}\right)$. En s'appuyant sur ces limitations, nous arrivons d'une façon connue à l'inégalité

$$
\left|D_{X}^{(m)}\left[\bar{W}_{\alpha \beta}(X, t ; Y, \tau)\right]\right|<\frac{\text { const. }}{(t-\tau)^{\mu+\mu_{1}-1}} \cdot \frac{1}{|X Y|^{n+m-M\left(\mu+\mu_{1}-1\right)-h_{1}}},
$$

si $|X Y| \leqq R_{0}$, et à l'inégalité

$$
\left|D_{X}{ }^{(m)}\left[\bar{W}_{\alpha \beta}(X, t ; Y, \tau)\right]\right|<\frac{\text { const. }(t-\tau)^{\mu^{\prime}}}{|X Y|^{n+m+M \mu^{\prime}}},
$$

si $|X Y|>R_{0}$. Nous en déduirons les limitations suivantes pour les dérivées d'ordre $m \leqq M-1$ des composantes des solutions fondamentales

$$
\left|D_{X}^{(m)}\left[\Gamma_{\alpha \beta}(X, t ; Y, \tau)\right]\right|<\frac{\text { const. }}{(t-\tau)^{\mu}} \cdot \frac{1}{|X Y|^{n+m-M \mu}},
$$

si $|X Y| \leqq R_{0}$, et

$$
\left|D_{X}{ }^{(m)}\left[\Gamma_{\alpha \beta}(X, t ; Y, \tau)\right]\right|<\frac{\text { const. }(t-\tau)^{\mu^{\prime}}}{|X Y|^{n+m+M \mu^{\prime}}},
$$

si $|X Y|>R_{0} ;\left(\mu^{\prime}>0\right)$. Les singularités de la limitation (31) seront faibles dans le cas $m<M$, si nous choisirons la constante $\mu$ à l'intérieur de l'intervalle

$$
m M^{-1}<\mu<1 \quad(m<M),
$$

si $n+m \geqq M$ et $m M^{-1}<\mu<(n+m) M^{-1}$, si $n+m<M$.

3. Intégrales analogues à l'intégrale de Poisson-Weierstrass. Soient les intégrales de volume

$$
J_{\alpha}(X, t, \tau)=\iiint_{\Omega} \sum_{\beta=1}^{N} \Gamma_{\alpha \beta}(X, t ; Y, \tau) \varrho_{\beta}(Y, \tau) d Y,
$$

où $\varrho_{\beta}(Y, \tau)$ sont des fonctions déterminées et intégrables dans la région fermée

$$
Y \in \Omega ; \quad 0 \leqq \tau \leqq T .
$$

Les fonctions (32) $(\alpha=1,2, \ldots, N)$ sont déterminées en tout point $X$ de l'espace $E$ pour $0<\tau<t<T$ et présentent une solution du système d'équations (1) dans cette région

$$
\hat{\Psi}^{(\alpha)}\left[J_{1}, J_{2}, \ldots, J_{N}\right]=0
$$

$(\alpha=1,2, \ldots, N)$. Les intégrales (32) sont analogues à l'intégrale de Poisson-Weierstrass dans la théorie de l'équation de chaleur.

Les intégrales (32) possèdent la propriété limite suivante. 
ThÉonème 1. Si les fonctions $\varrho_{\beta}(Y, \tau)$ sont continues dans la région fermée $\left(32^{\prime}\right)$, les intégrales (32) tendent vers les limites

$$
\lim _{\tau \rightarrow t} J_{\alpha}(X, t, \tau)=\varrho_{\alpha}(X, t)
$$

uniformément par rapport à $X$ en tout domaine fermé $\Omega^{*}$ situé à l'intérieur du domaine $\Omega$.

Démonstration. Écrivons, d'après la formule (25),

$$
J_{\alpha}(X, t, \tau)=J_{\alpha}^{\prime}(X, t, \tau)+J_{\alpha}^{\prime \prime}(X, t, \tau),
$$

où

$$
\begin{aligned}
& J_{\alpha}^{\prime}(X, t, \tau)=\iiint_{\Omega} \sum_{\beta=1}^{N} W_{\alpha \beta}^{Y, \tau}(X, t ; Y, \tau) \varrho_{\beta}(Y, \tau) d Y, \\
& J_{\alpha}^{\prime \prime}(X, t, \tau)=\iiint_{\Omega} \sum_{\beta=1}^{N} \bar{W}_{\alpha \beta}(X, t ; Y, \tau) \varrho_{\beta}(Y, \tau) d Y .
\end{aligned}
$$

La première intégrale $\left(34^{\prime}\right)$ était étudiée dans notre travail [5], où nous avons démontré la propriété limite

$$
\lim _{\tau \rightarrow t} J_{\alpha}^{\prime}(X, t, \tau)=\varrho_{\alpha}(X, t) \quad(\alpha=1,2, \ldots, N)
$$

la tendance vers la limite étant uniforme dans tout domaine fermé $\Omega^{*}$ situé à l'intérieur du domaine $\Omega$.

Pour étudier la seconde intégrale (34"), il suffit d'appliquer la limitation (27) des fonctions $\bar{W}$. Nous aurons alors

$$
\left|J_{\alpha}^{\prime \prime}(X, t, \tau)\right|<\frac{\text { const. } \sup \left|\varrho_{\beta}\right|}{(t-\tau)^{\mu+\mu_{1}-1}} \iiint_{\Omega} \frac{d Y}{|X Y|^{n-M\left(\mu+\mu_{1}-1\right)-h_{1}}},
$$

où $0<\mu<1,1-h_{1} M^{-1}<\mu_{1}<1$. Or, la constante $\mu_{1}$ étant fixée dans l'intervalle ouvert $\left(1-h_{1} M^{-1}, 1\right)$, on peut toujours choisir la constante positive $\mu$ suffisamment petite, pour qu'on ait

$$
\mu+\mu_{1}-1<0
$$

ensuite, d'après le choix de $\mu_{1}$, on a toujours

$$
n-M\left(\mu_{1}-1\right)-h_{1}<n,
$$

et l'intégrale de volume dans l'inégalité (36) est bornée. Nous en concluons la propriété limite 


$$
\lim _{\tau \rightarrow t} J_{\alpha}^{\prime \prime}(X, t, \tau)=0,
$$

la tendance étant uniforme dans tout le domaine $\Omega$.

Les résultats $(35)$ et $(37)$ confirme la thèse $(33)$ du théorème 1.

D'après la limitation $\left(28^{\prime}\right)$, la propriété limite (33) est vraie de même pour les intégrales de la forme (32), étendues en tout l'espace $E$, les fonctions $\varrho_{\beta}(Y, \tau)$ étant continues et bornées dans l'espace $E$.

Si les fonctions $\varrho_{\beta}(Y, \tau)$ n'étaient qu'intégrables dans le domaine $\Omega$, on ne peut pas affirmer l'existence de la limite (33), mais en tout cas, d'après la limitation (28), les intégrales (32) vérifient l'inégalité

$$
\left|J_{\alpha}(X, t, \tau)\right|<\frac{\text { const. } \sup \left|\varrho_{\beta}\right|}{(t-\tau)^{\mu}},
$$

l'exposant $\mu$ étant une constante positive, arbitrairement petit. Nous signalons encore, sous l'hypothèse que les fonctions $\varrho_{\beta}$ soient intégrables, que les dérivées spatiales d'ordre $m<M$ des intégrales (32), d'après les inégalités (31), admettent une limitation

$$
\left|D_{X}{ }^{(m)}\left[J_{\alpha}(X, t, \tau)\right]\right|<\frac{\text { const. sup }\left|\varrho_{\beta}\right|}{(t-\tau)^{\mu_{1}}}
$$

à faible singularité, si $\tau \rightarrow t$, la constante $\mu_{1}$ étant choisie à l'intérieur de l'intervalle $\left(m M^{-1}, 1\right)$, si $n+m \geqq M$ et à l'intérieur de l'intervalle $\left(m M^{-1},(n+m) M^{-1}\right)$, si $n+m<M$.

4. Potentiel de charge spatiale et ses dérivées. Nous appellerons potentiel de charge spatiale, relatif au système d'équations (1), une suite de $N$ fonctions $\left\{V_{\alpha}(X, t)\right\}$ données par les intégrales de volume

$$
V_{\alpha}(X, t)=\int_{0}^{t} \iiint_{\Omega} \sum_{\beta=1}^{N} \Gamma_{\alpha \beta}(X, t ; Y, \tau) \varrho_{\beta}(Y, \tau) d Y d \tau
$$

$(\alpha=1,2, \ldots, N)$. Les $N$ fonctions de la suite $\left\{\varrho_{\beta}(Y, \tau)\right\}$, dite densité de la charge, sont déterminées, bornées et intégrables dans la région bornée

$$
Y \in \Omega ; \quad 0 \leqq \tau \leqq T .
$$

D'après la continuité des fonctions $\Gamma_{\alpha \beta}$ et la limitation (28), les composantes (39) du potentiel sont continues dans tout l'espace $E$ pour $0 \leqq t \leqq T$ et vérifient les inégalités

$$
\left|V_{\alpha}(X, t)\right|<\text { const. } t^{1-\mu} \cdot \sup \left|\varrho_{\beta}\right|
$$

Donc ces composantes tendent vers zéro, si $t \rightarrow 0$. 
ThÉoRème 2. Si les composantes $\varrho_{\beta}(Y, \tau)$ de la densité sont bornées et intégrables dans la région (39'), les composantes (39) du potentiel de charge spatiale admettent les dérivées spatiales d'ordre $m \leqq M-1$ continues en tout point de l'espace $E$, données par les intégrales absolument convergentes

$$
D_{X}{ }^{(m)}\left[V_{\alpha}(X, t)\right]=\int_{0}^{t} \iiint_{\Omega} \sum_{\beta=1}^{N} D_{X}{ }^{(m)}\left[\Gamma_{\alpha \beta}(X, t ; Y, \tau)\right] \varrho_{\beta}(Y, \tau) d Y d \tau .
$$

La démonstration de ce théorème est analogue à la démonstration donnée pour le quasi-potentiel dans notre travail [2] et repose sur les limitations (31) aux faibles singularités, si $m \leqq M-1$.

Nous concluons encore que ces dérivées vérifient les inégalités de la forme

$$
\left|D_{X}^{(m)}\left[V_{\alpha}(X, t)\right]\right|<\text { const. } t^{1-\mu} \cdot \sup \left|\varrho_{\beta}\right|,
$$

$\sup \left|\varrho_{\beta}\right|$ désignant la plus grande des bornes supérieures des fonctions $\left|\varrho_{\beta}\right|, \mu$ est choisi à l'intérieur de l'intervalle $\left(m M^{-1}, 1\right)$; donc les dérivées (41) tendent vers zéro, si $t \rightarrow 0$.

L'existence des dérivées spatiales bornées jusqu'à l'ordre $M-1$ nous apprend que les fonctions (39) et leurs dérivées spatiales jusqu'à l'ordre $M-2$ vérifient la condition de Lipschitz par rapport aux variables $X\left(x_{1}, \ldots, x_{n}\right)$ dans le domaine $[\Omega ;(0, T)]$. Intégrabilité des fonctions $\varrho_{\beta}(Y, \tau) n$ 'est pas suffisante pour en conclure l'existence des dérivées des fonctions (39) et (41) par rapport à la variable $t$ à l'intérieur du domaine $\Omega$, mais nous avons alors le théorème suivant.

ThÉORÈme 3. Si les fonctions de la densité $\left\{\varrho_{\beta}(Y, \tau)\right\}$ sont bornées et intégrables dans le domaine $[\Omega ;(0, T)]$, les composantes (39) du potentiel de charge spatiale et leurs dérivées spatiales d'ordre $m<M$ vérifient dans le domaine $[\Omega ;(0, T)]$ les conditions de Hölder relativement à la variable $t$ des formes suivantes

$$
\begin{gathered}
\left|V_{\alpha}(X, t)-V_{\alpha}\left(X, t_{1}\right)\right|<\text { const. } \sup \left|\varrho_{\beta}\right| \cdot\left|t-t_{1}\right|^{\theta} \\
\left|D_{X}{ }^{(m)}\left[V_{\alpha}(X, t)\right]-D_{X}{ }^{(m)}\left[V_{\alpha}\left(X, t_{1}\right)\right]\right|<\text { const. } \sup \left|\varrho_{\beta}\right| \cdot\left|t-t_{1}\right|^{\theta\left(1-m M^{-1}\right)}
\end{gathered}
$$
$(m=0,1, \ldots, M-1 ; \alpha=1,2, \ldots, N), \theta$ désignant une constante positive arbitraire, inférieure d̀ l'unité, si $m+n \geqq M$ et $0<\theta<n(M-m)^{-1}$, si $m+n<M$, les coefficients constants positifs ne dépendent des fonctions $\varrho_{\beta}$.

Démonstration. Nous ne démontrerons que la propriété (44), la démonstration de la propriété (43) est analogue et plus facile. D'après la formule (21), nous pouvons exprimer les composantes (39) du potentiel de charge spatiale sous la forme des sommes 


$$
V_{\alpha}(X, t)=V_{\alpha}^{*}(X, t)+V_{\alpha}^{* *}(X, t)
$$

des composantes des deux quasi-potentiels

$$
\begin{aligned}
V_{\alpha}^{*}(X, t) & =\int_{0}^{t} \iiint_{\Omega(Y)} \sum_{\beta=1}^{N} W_{\alpha \beta}^{Y, \tau}(X, t ; Y, \tau) \varrho_{\beta}(Y, \tau) d Y d \tau, \\
V^{* *}(X, t) & =\int_{0}^{t} \iiint_{E(I)} \sum_{\gamma=1}^{N} W_{\alpha \gamma}^{\Pi, \xi}(X, t ; \Pi, \zeta) \bar{\varrho}_{\gamma}(\Pi, \zeta) d \Pi d \zeta,
\end{aligned}
$$

de densité donnée $\left\{\varrho_{\beta}(Y, \tau)\right\}$ et de densité $\left\{\bar{\varrho}_{\gamma}(\Pi, \zeta)\right\}$ exprimée par les formules

$$
\bar{\varrho}_{\gamma}(\Pi, \zeta)=\int_{0}^{\zeta} \iint_{\Omega(Y)} \int_{\beta=1} \sum_{\beta \beta}^{N} \Phi_{\alpha \beta}(\Pi, \zeta ; Y, \tau) \varrho_{\beta}(Y, \tau) d Y d \tau .
$$

D'après les propriétés $(24)$ et $\left(24^{\prime}\right)$, les fonctions (48) sont continues et bornées en tout l'espace $E$ pour $0 \leqq \zeta \leqq T$.

Étudions d'abord les dérivées spatiales des fonctions (46). Écrivons donc, en supposant $t_{1}<t$,

$$
\begin{aligned}
& D_{X}{ }^{(m)}\left[V_{\alpha}^{*}(X, t)\right]-D_{X}^{(m)}\left[V_{\alpha}^{*}\left(X, t_{1}\right)\right] \\
= & \int_{i_{1}}^{t} \iiint_{\Omega(Y)} \sum_{\beta=1}^{N} D_{X}^{(m)}\left[W^{Y, \tau}(X, t ; Y, \tau)\right] \varrho_{\beta}(Y, \tau) d Y d \tau+ \\
+ & \int_{0}^{t_{1}} \iiint_{\Omega(Y} \int_{\beta=1} \sum_{\beta=1}^{N}\left\{D_{X}^{(m)}\left[W^{Y, \tau}(X, t ; Y, \tau)\right]-D_{X}^{(m)}\left[W^{Y, \tau}\left(X, t_{1} ; Y, \tau\right)\right]\right\} \varrho_{\beta}(Y, \tau) d Y d \tau .
\end{aligned}
$$

La première intégrale, $J_{1}$, de la somme (49), d'après la limitation (20), vérifie l'inégalité

$$
\left|J_{1}\right|<\text { const. } \sup \left|\varrho_{\beta}\right| \cdot\left|t-t_{1}\right|^{1-\mu},
$$

où $\mu$ est une constante arbitrairement choisie dans l'intervalle ouvert $\left(m M^{-1}, 1\right)$ si $n+m \geqq M$ et dans l'intervalle ouvert $\left(m M^{-1},(n+m) M^{-1}\right)$ si $n+m<M$, la constante positive ne depend des fonctions $\varrho_{\beta}$.

La seconde intégrale, $J_{2}$, dans la somme (49) nous décomposons en deux intégrales

$$
J_{2}=J_{2}^{\Delta^{\prime}}+J_{2}^{\Omega-\Delta^{\prime}}
$$

étendues au domains $\Delta^{\prime}=\Delta \times \Omega$ (commun au domaine $\Omega$ et à la sphère $\Delta$ de rayon $r_{\Delta}$, de centre $X$ ) et au domaine extérieure $\Omega-\Delta^{\prime}$. Remarque faite de la limitation (20), nous aurons 


$$
\left|J_{2}^{\Delta^{\prime}}\right|<\mathrm{const} . \int_{0}^{t_{1}} \frac{\sup \left|\varrho_{\beta}\right| d \tau}{\left(t_{1}-\tau\right)^{\mu}} \iiint_{\Delta} \frac{d Y}{|X Y|^{n+m-M \mu_{2}}}<\text { const. } \sup \left|\varrho_{\beta}\right| \cdot r_{\Delta}^{M \mu_{2}-m}
$$
où $m M^{-1}<\mu_{2}<1$, si $m+n \geqq M$ et $m M^{-1}<\mu_{2}<(m+n) M^{-1}$, si $m+n<M$.

Pour limiter la seconde partie de la somme (51), remarquons que la dérivée par rapport à la variable $t$ de la dérivée spatiale d'ordre $m$ de la fonction $W_{\alpha \beta}$ admet, d'aprés (10), la limitation de la même forme que la dérivée spatiale d'ordre $M+m$ de la fonction $W_{\alpha \beta}$, nous aurons donc, en tenant compte de l'inégalité (20),

$$
\left|D_{X t^{(m+1)}}\left[W_{\alpha \beta}^{Y, \tau}(X, t ; Y, \tau)\right]\right|<\frac{\text { const. }}{(t-\tau)^{\mu_{3}}} \frac{1}{|X Y|^{n+m+M\left(1-\mu_{3}\right)}}
$$

$\left(0<\mu_{3}<1\right)$. Nous en concluons

$$
\begin{aligned}
\left|J_{2}^{\Omega-\Delta^{\prime}}\right| & <\text { const. } \sup \left|\varrho_{\beta}\right| \cdot\left|t-t_{1}\right| \iiint_{\Omega-d^{\prime}} \frac{d Y}{|X Y|^{n+m+M\left(1-\mu_{3}\right)}} \\
& <\text { const. } \sup \left|\varrho_{\beta}\right| \cdot\left|t-t_{1}\right|\left[r_{\Delta}^{M\left(\mu_{3}-1\right)-m}+L^{M\left(\mu_{3}-1\right)-m}\right],
\end{aligned}
$$

$L$ désignant le diamètre du domaine $\Omega$. Nous posons maintenant

$$
r_{\Delta}=\text { const. }\left|t-t_{1}\right|^{\alpha}
$$

en choisissant la constante positive $\alpha$ de façon que

$$
1-\alpha\left[M\left(1-\mu_{3}\right)+m\right]=\left(M \mu_{2}-m\right) \alpha>0, \quad \mu_{2}=\mu_{3},
$$

et nous aurons $\alpha=1 / M$. Il en résulte que la différence des dérivées (49) vérifie l'inégalité de Hölder

$$
\left|D_{X}^{(m)}\left[V_{\alpha}^{*}(X, t)\right]-D_{X}^{(m)}\left[V_{\alpha}^{*}\left(X, t_{1}\right)\right]\right|<\text { const. } \sup _{\Omega}\left|\varrho_{\beta}\right| \cdot\left|t-t_{1}\right|^{\theta\left(1-m M^{-1}\right)},
$$

où $\theta$ désigne une constante positive arbitraire, inférieure à l'unité, si $m+n \geqq M$ et $0<\theta<n(M-m)^{-1}$, si $m+n<M$.

D'une façon tout pareille on étudiera les dérivées des intégrales (47) étendues à tout l'espace $E$ avec telle différence que maintenant, pour obtenir une limitation de l'intégrale étendue au domaine $E-\Delta$, il faut profiter des inégalités $(20)$ et $\left(20^{\prime}\right)$. En remarquant encore que la fonction (48) vérifie l'inégalité

$$
\sup _{E}\left|\bar{\varrho}_{\gamma}(\Pi, \zeta)\right|<\text { const. } \zeta^{1-\mu} \cdot \sup _{\Omega}\left|\varrho_{\beta}(Y, \tau)\right|
$$

nous arriverons aux inégalités pour la différence des dérivées d'ordre $m<M$ des fonctions $V_{\alpha}^{* *}(X, t)$ de la même forme que (55). Il en résulte la thèse $(44)$ du théorème 3 . 
Si les composantes de la densité $\left\{\varrho_{\beta}(Y, \tau)\right\}$ ne sont que bornées et intégrables, nous ne pouvons en conclure l'existence des dérivées spatiales d'ordre $M$ du potentiel (39), mais nous avons le théorème suivant.

ThÉonÈme 4. Si les fonctions $\varrho_{\beta}(Y, \tau)$ sont bornées et intégrables dans le domaine $[\Omega ;(0, T)]$, les dérivées spatiales d'ordre $M-1$ des composantes (39) $d u$ potentiel de charge spatiale vérifient dans le domaine $[\Omega ;(0, T)]$ la condition de Hölder par rapport aux variables spatiales

$$
\left|D_{X}^{(M-1)}\left[V_{\alpha}(X, t)\right]-D_{X}^{(M-1)}\left[V_{\alpha}\left(X_{1}, t\right)\right]\right|<\text { const. } \sup _{\Omega}\left|\varrho_{\beta}\right| \cdot\left|X X_{1}\right|^{\theta},
$$

$\theta$ désignant un nombre positif arbitraire, inférieur à l'unité.

Démonstration. De même que précédemment, nous décomposons les fonctions (39) en sommes des intégrales (46) et (47) et nous étudierons les intégrales (46). Écrivons donc

$$
\begin{gathered}
D_{X}^{(M-1)}\left[V_{\alpha}(X, t)\right]-D_{X}^{(M-1)}\left[V_{\alpha}\left(X_{1}, t\right)\right] \\
=\int_{0}^{t} \iiint_{\Omega(Y)} \sum_{\beta=1}^{N}\left\{D_{X}^{(M-1)}\left[W_{\alpha \beta}^{Y, \tau}(X, t ; Y, \tau)\right]-D_{X}^{(M-1)}\left[W_{\alpha \beta}^{Y, \tau}\left(X_{1}, t ; Y, \tau\right)\right]\right\} \varrho_{\beta}(Y, \tau) d Y d \tau \\
=I^{K^{\prime}+I^{\Omega-K^{\prime}},}
\end{gathered}
$$

où $I^{K^{\prime}}$ désigne l'intégrale étendue au domaine $K^{\prime}$ commun au domaine $\Omega$ et à la sphère $K$ de centre $X$ et de rayon $2\left|X X_{1}\right|, I^{\Omega-K^{\prime}}$ désigne l'intégrale (57) étendue au domaine extérieur $\Omega-K^{\prime}$. D'après la limitation (20), nous aurons

$$
\begin{aligned}
\left|I^{K^{\prime}}\right| & <\text { const. } \sup \left|\varrho_{\beta}\right| \int_{0}^{3\left|X X_{1}\right|} r^{M \mu-M} d r \\
& =\text { const. } \sup \left|\varrho_{\beta}\right| \cdot\left|X X_{1}\right|^{M \mu-M+1} \quad\left(1-M^{-1}<\mu<1\right) .
\end{aligned}
$$

Pour limiter l'intégrale $I^{\Omega-K^{\prime}}$, nous écrivons, d'après le théorème des accroissements,

$$
\begin{aligned}
& D_{X}{ }^{(M-1)}\left[W_{\alpha \beta}^{Y, \tau}(X, t ; Y, \tau)\right]-D_{X}{ }^{(M-1)}\left[W_{\alpha \beta}^{Y, \tau}\left(X_{1}, t ; Y, \tau\right)\right] \\
& =\sum_{\nu=1}^{N}\left\{\frac{\partial}{\partial x_{v}} D_{X}^{(M-1)}\left[W_{\alpha \beta}^{Y, \tau}(X, t ; Y, \tau)\right]\right\}_{X_{*}}\left(x_{\nu}-x_{\nu}^{\prime}\right),
\end{aligned}
$$

où $\left(x_{1}^{\prime}, \ldots, x_{n}^{\prime}\right)$ désignent les coordonnées du point $X_{1}$ et $X_{*}$ désigne un point du segment $X X_{1}$. Remarque faite de la limitation (20) pour $m=M$ et de l'inégalité

$$
\left|X_{*} Y\right| /|X Y|>\frac{1}{2},
$$

si $Y \in \Omega-K$, nous aurons l'inégalité 
(59)

$$
\begin{gathered}
\left|D_{X}^{(M-1)}\left[W_{\alpha \beta}^{Y, \tau}(X, t ; Y, \tau)\right]-D_{X}^{(M-1)}\left[W_{\alpha \beta}^{Y, \tau}\left(X_{1}, t ; Y, \tau\right)\right]\right| \\
<\frac{\text { const. }}{(t-\tau)^{\mu}} \cdot \frac{\left|X X_{1}\right|}{|X Y|^{n+M(1-\mu)}}
\end{gathered}
$$

vraie, si $Y \in \Omega-K$. Il en résulte la limitation

$$
\begin{aligned}
\left|I^{\Omega-K^{\prime}}\right| & <\text { const. } \sup \left|\varrho_{\beta}\right| \cdot\left|X X_{1}\right| \iiint_{\Omega-K} \frac{d Y}{|X Y|^{n+M\left(1-\mu_{1}\right)}} \\
& <\text { const. } \sup \left|\varrho_{\beta}\right| \cdot\left|X X_{1}\right| \cdot \int_{2\left|X X_{1}\right|}^{L} \frac{d r}{r^{M(1-\mu)+1}} \\
& <\text { const. } \sup \left|\varrho_{\beta}\right| \cdot\left|X X_{1}\right|^{1-M(1-\mu)} \quad\left(1-M^{-1}<\mu<1\right) .
\end{aligned}
$$

En rapprochant les résultats (58) et (60), nous en concluons la limitation

$$
\left|D_{X}^{(M-1)}\left[V_{\alpha}(X, t)\right]-D_{X}^{(M-1)}\left[V_{\alpha}\left(X_{1}, t\right)\right]\right|<\text { const. } \sup \left|\varrho_{\beta}\right| \cdot\left|X X_{1}\right|^{\theta}
$$

$\theta$ étant une constante positive arbitraire, inférieure à l'unité. D'une façon tout pareille on étudiera la différence des dérivées des intégrales (47) et one arrivera à la thèse (56) du théorème 4.

5. Etude des dérivées d'ordre $M$ du potentiel. Si $m=M$, on ne peut pas choisir la constante positive $\mu$ dans la limitation (31), pour qu'on ait à la fois $0<\mu<1$ et $n+m-M \mu<n$.

La recherche des dérivées spatiales d'ordre $M$ (égal à l'ordre des équations (1)) des composantes (39) du potentiel exige donc des considérations plus délicates que pour les dérivées d'ordre $m<M$. Nous avons démontré dans le travail [5] que si les composantes de la densité $\varrho_{\beta}(Y, \tau)$ vérifient une condition de Hölder

$$
\left|\varrho_{\beta}(Y, \tau)-\varrho_{\beta}\left(Y_{1}, \tau\right)\right|<\text { const. }\left|Y Y_{1}\right|^{h_{\varrho}}
$$

$\left(0<h_{\varrho} \leqq 1\right)$ et sont continues par rapport à la variable $\tau$ dans le domaine $[Y \in \Omega ; 0 \leqq \tau \leqq T]$, alors les composantes (46) du quasi-potentiel admettent les dérivées d'ordre $M$, données par les intégrales absolument convergentes

$$
D_{X}(M)\left[V_{\alpha}^{*}(X, t)\right]=\int_{0}^{t} D_{X}^{(M)}\left[J_{\alpha}^{\prime}(X, t, \tau)\right] d \tau,
$$

où la fonction $J_{\alpha}^{\prime}$ est donnée par $\left(34^{\prime}\right)$ et $1^{\prime}$ on a 


$$
\begin{aligned}
& D_{X}^{(M)}\left[J_{\alpha}^{\prime}(X, t, \tau)\right] \\
& =\sum_{\beta=1}^{N} \varrho_{\beta}(X, \tau)\left\{-\iint_{K^{\prime}} D_{X}{ }^{(M-1)}\left[W_{\alpha \dot{\beta}}^{P, \tau}(X, t ; Q, \tau)\right] \cos v_{j} d \sigma_{Q}+\right. \\
& \left.\quad+\iiint_{\Omega} D_{X} D_{X}^{(M)}\left[W_{\alpha \beta}^{P, \tau}(X, t ; Y, \tau)\right] d Y\right\}_{P=X}+ \\
& +\sum_{\beta=1}^{N} \varrho_{\beta}(X, \tau) \iiint_{\Omega}\left\{D_{X}^{(M)}\left[W_{\alpha \beta}^{Y, \tau}(X, t ; Y, \tau)\right]-\right. \\
& \left.\quad-\left(D_{X}{ }^{(M)}\left[W_{\alpha \beta}^{P, \tau}(X, t ; Y, \tau)\right]\right)_{P=X}\right\} d Y+ \\
& +\iiint_{\Omega} \sum_{\beta=1}^{N} D_{X}{ }^{(M)}\left[W_{\alpha \beta}^{Y, \tau}(X, t ; Y, \tau)\right]\left[\varrho_{\beta}(Y, \tau)-\varrho_{\beta}(X, \tau)\right] d Y,
\end{aligned}
$$

où $K^{\prime}$ désigne la surface de la sphère $K$, de centre fixé $X_{0}$, située à l'intérieur du domaine $\Omega$ et contenant le point $X$ à son intérieur; $v_{j}$ désigne l'angle que fait la normale à la surface $K^{\prime}$ avec l'axe d'une telle variable $x_{j}$ par rapport à laquelle on a différentié, en passant de la dérivée $D_{X}{ }^{(M-1)}$ à la dérivée $D_{X}{ }^{(M)}$. Nous avons démontré (voir [5]) que la somme (64) vérifie l'inégalité à faible singularité

$$
\left|D_{X}^{(M)}\left[J^{\prime}(X, t, \tau)\right]\right|<\frac{C_{X_{0}}}{(t-\tau)^{\mu}},
$$

$\mu$ étant choisi à l'intérieur de l'intervalle

$$
1-h_{*} M^{-1}<\mu<1,
$$

où $h_{*}=\min \left(h, h_{\varrho}\right) ; C_{X_{0}}$ est une constante positive fixée au voisinage du point $X_{0}$.

D'après les formules $\left(34^{\prime}\right)$, nous pouvons écrire aussi

$$
D_{X}{ }^{(M)}\left[V_{\alpha}^{*}(X, t)\right]=\int_{0}^{t} \iiint_{\Omega} \sum_{\beta=1}^{N} D_{X}{ }^{(M)}\left[W_{\alpha \beta}^{Y, \tau}(X, t ; Y, \tau)\right] \varrho_{\beta}(Y, \tau) d Y d \tau ;
$$

cette intégrale n'est pas, en général, absolument convergente.

Il reste à étudier les dérivées d'ordre $M$ des intégrales (47) qui ont la forme des composantes du quasi-potentiel de densité (48). Dans ce but nous démontrerons d'abord le théorème auxiliaire suivant.

ThÉoRìme AUXILIAIRE. Si les fonctions $\varrho_{\beta}(Y, \tau)$ sont bornées et intégrables dans la région $[Y \in \Omega ; 0 \leqq \tau \leqq T]$, les fonctions (48) vérifient dans. cette région une condition de Hölder de la forme

$$
\left|\bar{\varrho}_{\gamma}\left(\Pi_{1}, \zeta\right)-\bar{\varrho}_{\gamma}(\Pi, \zeta)\right|<\text { const. sup }\left|\varrho_{\beta}\right| \cdot\left|\Pi \Pi_{1}\right|^{\theta h_{1}},
$$


où $h_{1}=\min \left(h, 2 h^{\prime}\right), \theta$ est une constante positive arbitraire, inférieure à l'unité.

Démonstration. Les $N$ solutions du système d'équations intégrales (22) sont données par les formules

$$
\begin{aligned}
\Phi_{\alpha \beta}(X, t ; Y, \tau)= & f_{\alpha \beta}(X, t ; Y, \tau)+ \\
& +\int_{\tau}^{t} \iiint_{E} \int_{\nu=1}^{N} \Re_{\alpha \gamma}(X, t ; \Pi, \zeta) f_{\alpha \beta}(\Pi, \zeta ; Y, \tau) d Y d \tau,
\end{aligned}
$$

où les noyaux résolvants $\mathfrak{R}_{\alpha \gamma}$, étant les sommes de séries absoluments convergents (voir [5])

$$
\mathfrak{\Re}_{\alpha \gamma}(X, t ; Y, \tau)=\sum_{\nu=0}^{\infty} N_{\alpha \gamma}^{(\nu)}(X, t ; \Pi, \zeta)
$$

$\left(N_{\alpha \gamma}^{(0)}=N_{\alpha \gamma}\right)$, sont déterminées par les relations de récurrence

On a posé

$$
N_{\alpha \gamma}^{(v+1)}(X, t ; \Pi, \zeta)=\sum_{\delta=1}^{N} \iint_{\xi}^{t} \iiint_{E} N_{\alpha \delta}(X, t ; Z, s) N_{\delta \gamma}^{(v)}(Z, s ; \Pi, \zeta) d Z d s .
$$

$$
\begin{aligned}
& N_{\alpha \gamma}(X, t ; \Pi, \zeta)=\hat{\Psi}_{X, t}^{(\alpha)}\left[W_{1 \gamma}^{I I, \zeta} ; W_{2 \gamma}^{I I, \zeta} ; \ldots ; W_{N \gamma}^{I, \zeta}\right] \\
& \quad=\sum_{1 \leqq j \leqq N}\left[A_{\alpha j}^{k_{1} \ldots k_{n}}(X, t)-A_{\alpha j}^{k_{1} \ldots k_{n}}(\Pi, \zeta)\right] \frac{\partial^{M} W_{j \gamma}^{I I, \zeta}(X, t ; \Pi, \zeta)}{\partial x_{1}^{k_{1}} \ldots \partial x_{n}{ }^{k_{n}}}+ \\
& \quad+\sum_{1 \leqq k_{1}+\ldots+k_{n}<M} B_{\alpha j}^{k_{1} \ldots k_{n}}(X, t) \frac{\partial^{k_{1}+\ldots+k_{n}}}{\partial x_{1}{ }^{k_{1}} \ldots \partial x_{n}{ }^{k_{n}}}\left[W_{j \gamma}^{I,, \zeta}(X, t ; \Pi, \zeta)\right]+ \\
& \quad+\sum_{1 \leqq j \leqq N} C_{\alpha j}(X, t) W_{j \gamma}^{I, \zeta}(X, t ; \Pi, \zeta) .
\end{aligned}
$$

Nous avons démontré, en s'appuyant sur les inégalités (3) et (20), que les fonctions (72) admettent une limitation à faible singularité de la même forme que (24) et $\left(24^{\prime}\right)$ :

$$
\begin{array}{r}
\left|N_{\alpha \beta}(X, t ; Y, \tau)\right|<\frac{\text { const. }}{(t-\tau)^{\mu_{1}}} \frac{1}{|X Y|^{n+M\left(1-\mu_{1}\right)-h_{1}}} \\
\quad\left(1-h_{1} M^{-1}<\mu_{1}<1\right) .
\end{array}
$$

D'après l'égalité (69), nous décomposons les fonctions (48) en sommes des deux termes, dont le premier a la forme

$$
\bar{\varrho}_{\gamma}^{\prime}(\Pi, \zeta)=\int_{0}^{\zeta} \iint_{\Omega(Y)} \int_{\int^{\prime}} \sum_{\beta=1}^{N} f_{\gamma \beta}(\Pi, \zeta ; Y, \tau) \varrho_{\beta}(Y, \tau) d Y d \tau \text {. }
$$


Il suffit d'étudier le terme (73), puisque l'étude du second terme est analogique. Soit donc une sphère $K$ de centre $\Pi$ et de rayon $2\left|\Pi \Pi_{1}\right|$, $\Pi$ et $\Pi_{1}$ étant deux points arbitraires du domaine $\Omega$. Nous décomposons la différence des intégrales (73) en sommes des intégrales

$$
\begin{aligned}
& \bar{\varrho}_{\gamma}^{\prime}(\Pi, \zeta)-\bar{\varrho}_{\gamma}^{\prime}\left(\Pi_{1}, \zeta\right) \\
& =\int_{0}^{\zeta} \iiint_{K^{\prime}} \sum_{\beta=1}^{N}\left[f_{\gamma \beta}(\Pi, \zeta ; Y, \tau)-f_{\gamma \beta}\left(\Pi_{1}, \zeta ; Y, \tau\right)\right] \varrho_{\beta}(Y, \tau) d Y d \tau+ \\
& \quad+\int_{0}^{\zeta} \iiint_{\Omega} \int_{K^{\prime}} \sum_{\beta=1}^{N}\left[f_{\gamma \beta}(\Pi, \zeta ; Y, \tau)-f_{\gamma \beta}\left(\Pi_{1}, \zeta ; Y, \tau\right)\right] \varrho_{\beta}(Y, \tau) d Y d \tau
\end{aligned}
$$

étendues au domaine $K^{\prime}=\Omega \times K$ et au domains $\Omega-K^{\prime}$ extérieur à la sphère $K$. La première des intégrales (74), que nous désignerons par $I_{e}^{K^{\prime}}$, vérifie, d'après la limitation (24), l'inégalité

$$
\begin{aligned}
\left|I_{\varrho}^{K^{\prime}}\right| & <\text { const. } \sup \left|\varrho_{\beta}\right| \cdot \int_{0}^{3\left|\Pi \Pi_{1}\right|} r^{h_{1}-M\left(1-\mu_{1}\right)-1} d r \\
& =\text { const. } \sup \left|\varrho_{\beta}\right| \cdot\left|\Pi \Pi_{1}\right|^{h_{1}-M\left(1-\mu_{1}\right)} .
\end{aligned}
$$

Pour limiter la seconde des intégrales (74), que nous désignerons par $I_{\varrho}^{\Omega-K^{\prime}}$, nous allons chercher d'abord la limitation des différences sous le signe de l'intégrale.

En s'appuyant sur les inégalités admises (3), (4), sur les limitations (20) et analogues aux inégalités (59), nous obtenons, d'après la formule (72),

$$
\begin{aligned}
& \left|f_{\gamma \beta}(\Pi, \zeta ; Y, \tau)-f_{\gamma \beta}\left(\Pi_{1}, \zeta ; Y, \tau\right)\right|<\frac{\text { const. }}{(\zeta-\tau)^{\mu}} \frac{\left|\Pi \Pi_{1}\right|^{h}}{|\Pi Y|^{n+M-M_{\mu}}}+ \\
& \quad+\frac{\text { const. }}{(\zeta-\tau)^{\mu_{0}}} \cdot \frac{\left|\Pi \Pi_{1}\right|}{|\Pi Y|^{n+(M+1)\left(1-\mu_{0}\right)-h}}+\frac{\text { const. }}{(\zeta-\tau)^{\mu_{0}^{\prime}}} \cdot \frac{\left|\Pi \Pi_{1}\right|}{|\Pi Y|^{n+(M+1)\left(1-\mu_{0}^{\prime}-\bar{h}^{\prime}\right)}}
\end{aligned}
$$

en profitant de l'inégalité $\left|\Pi_{1} Y\right| /|\Pi Y|>\frac{1}{2}$, si $Y \in \Omega-K ; \mu, \mu_{0}, \mu_{0}^{\prime}$ sont des constantes positives arbitraires, inférieures à l'unité. Il en résulte

$$
\begin{aligned}
\left|I_{\varrho}^{\Omega-K^{\prime}}\right|<\text { const. } \sup & \left|\varrho_{\beta}\right| \cdot\left[\left|\Pi \Pi_{1}\right|^{h-M(1-\mu)}+\right. \\
& \left.+\left|\Pi \Pi_{1}\right|^{1+h-(M+1)\left(1-\mu_{0}\right)}+\left|\Pi \Pi_{1}\right|^{1-(M+1)\left(1-\mu^{\prime} 0-h^{\prime}\right)}\right] .
\end{aligned}
$$

En choisissant les constantes arbitraires $\mu, \mu_{0}, \mu_{0}^{\prime}$ dans les intervalles

(78) $1-\frac{h}{M}<\mu<1 ; \quad 1-\frac{h+1}{M+1}<\mu_{0}<1 ; \quad 1-h^{\prime}-\frac{1}{M+1}<\mu_{0}^{\prime}<1$ 
nous concluons la limitations

$$
\left|I_{\varrho}^{\Omega-K^{\prime}}\right|<\text { const. sup }\left|\varrho_{\beta}\right| \cdot\left|\Pi \Pi_{1}\right|^{\theta h}
$$

$(0<\theta<1)$. En réunissant les résultats $(75)$ et $(79)$, nous voyons que l'intégrale (73) vérifie la condition de Hölder de la forme

$$
\left|\varrho_{\gamma}^{\prime}(\Pi, \zeta)-\bar{\varrho}_{\gamma}^{\prime}\left(\Pi_{1}, \zeta\right)\right|<\text { const. sup }\left|\varrho_{\beta}\right| \cdot\left|\Pi \Pi_{1}\right|^{\theta h_{1}},
$$

$\theta$ étant une constante positive arbitraire, inférieure à l'unité. L'étude analogique de la seconde sommande de l'intégrale (48) fournit la condition de Hölder avec l'exposant non inférieur à l'exposant (80), il en résulte la thèse $(68)$ du théorème auxiliaire.

ThÉORÈme 5. Si les coefficients des équations (1) vérifient les conditions I, II, III et les composantes de la densité $\left\{\varrho_{\beta}(Y, \tau)\right\}$ - la condition de Hölder par rapport aux variables spatiales

$$
\left|\varrho_{\beta}(Y, \tau)-\varrho\left(Y_{1}, \tau\right)\right|<\text { const. }\left|Y Y_{1}\right|^{h_{\varrho}}
$$

$\left(0<h_{\varrho} \leqq 1\right)$ et sont continues par rapport à la variable $t$, alors les composantes du potentiel de charge spatiale (39) admettent les dérivées spatiales d'ordre $M$, en tout point intérieur $X \in \Omega$ pour $0<t<T$, données par les formules

$$
\begin{aligned}
D_{X}^{(M)}\left[V_{\alpha}(X, t)\right] & =\int_{0}^{t} \iiint_{\Omega(Y)} \sum_{\beta=1}^{N} D_{X}^{(M)}\left[W_{\alpha \beta}^{Y, \tau}(X, t ; Y, \tau)\right] \varrho_{\beta}(Y, \tau) d Y d \tau+ \\
& +\int_{0}^{t} \iiint_{E(I)} \sum_{\gamma=1}^{N} D_{X}^{(M)}\left[W_{\alpha \gamma}^{\Pi, \zeta}(X, t ; \Pi, \zeta)\right] \bar{\varrho}_{\gamma}(\Pi, \zeta) d \Pi d \zeta,
\end{aligned}
$$

où les intégrales ne sont pas, en général, absolument convergentes.

Démonstration. La formule (82) résulte de la formule (63) pour les dérivées d'ordre $M$ du quasi potentiel, résultant de la décomposition (64) et de la limitation (65) à faible singularité. En outre il faut s'appuyer sur le théorème (68). Nous signalons encore que la seconde des intégrales (82) a bien le sens, d'après la limitation $\left(20^{\prime}\right)$ des dérivées des quasi-solutions et l'inégalité $\left(55^{\prime}\right)$.

ThÉonÈme 6. Si les conditions du théorème 5 sont satisfaites, les composantes du potentiel (39) admettent les dérivées par rapport à la variable $t$ données par la formule 
(83) $\frac{\partial}{\partial t}\left[V_{\alpha}(X, t)\right]$

$$
\begin{aligned}
= & \varrho_{\alpha}(X, t)+\int_{0}^{t} \iiint_{\Omega(Y)} \sum_{\beta=1}^{N} \frac{\partial}{\partial t}\left[W_{\alpha \beta}^{Y, \tau}(X, t ; Y, \tau)\right] \varrho_{\beta}(Y, \tau) d Y d \tau+ \\
& +\bar{\varrho}_{\alpha}(X, t)+\int_{0}^{t} \iiint_{E(\Omega)} \sum_{\gamma=1}^{N} \frac{\partial}{\partial t}\left[W_{\alpha \gamma}^{\Pi, \zeta}(X, t ; \Pi, \zeta)\right] \bar{\varrho}_{\gamma}(\Pi, \zeta) d \Pi d \zeta
\end{aligned}
$$

en tout point intérieur $X$ du domaine $\Omega$, pour $0<t<T$.

Démonstration. Le premier (46) des deux quasi-potentiels (45) vérifie l'équation aux dérivées partielles (16), où il faut remplacer $Z, \zeta$ par $Y, \tau$. Donc l'intégrale régulière $(\tau<t)$

$$
\frac{\partial}{\partial t}\left[J_{\alpha}^{\prime}(X, t, \tau)\right]=\iiint_{\Omega} \sum_{\beta=1}^{N} \frac{\partial}{\partial t}\left[W_{\alpha \beta}^{Y, \tau}(X, t ; Y, \tau)\right] \varrho_{\beta}(Y, \tau) d Y,
$$

d'après la limitation (65), vérifie l'inégalité à faible singularité

$$
\left|\frac{\partial}{\partial t}\left[J_{\alpha}^{\prime}(X, t, \tau)\right]\right|<\frac{\text { const. }}{(t-\tau)^{\mu}}
$$

où $1-h_{*} M^{-1}<\mu<1 ; h_{*}=\min \left(h, h_{\varrho}\right)$, le point $X$ étant fixé à l'intérieur du domaine $\Omega$.

En s'appuyant sur cette propriété et sur la propriété limite (35), on peut montrer d'une façon analogue que dans notre travail [4] (pages 37-40), que la dérivée par rapport à la variable $t$ de la composante (46) du quasi potentiel s'exprime comme il suit

$$
\begin{aligned}
\frac{\partial}{\partial t}\left[V_{\alpha}^{*}(X, t)\right] & =\lim _{\tau \rightarrow t} J_{\alpha}^{\prime}(X, t, \tau)+\int_{0}^{t} \frac{\partial}{\partial t}\left[J_{\alpha}^{\prime}(X, t, \tau)\right] d \tau \\
& =\varrho_{\alpha}(X, t)+\int_{0}^{t} \frac{\partial}{\partial t}\left[J_{\alpha}^{\prime}(X, t, \tau)\right] d \tau,
\end{aligned}
$$

ce qui est en accord avec la règle classique pour les intégrales régulières. On obtiendra la formule analogue pour la deuxième intégrale (47) et on arrivera à la formule (83), où la fonction $\bar{\varrho}_{\alpha}$ est donnée par l'expression (48).

ThúonÈme 7. Si les conditions du théorème 5 sont satisfaites, le potentiel de charge spatiale $\left\{V_{\alpha}(X, t)\right\}$, donné par les formules (39), vérifie le système d'équations 


$$
\hat{\Psi}^{(\alpha)}\left[V_{1}(X, t), \ldots, V_{N}(X, t)\right]=-\varrho_{\alpha}(X, t)
$$

$(\alpha=1,2, \ldots, N)$ en tout point intérieur $X$ du domaine $\Omega$ pour $0<t<T$; $\hat{\Psi}^{(a)}$ désignent les opérations différentielles, définies par les formules (1).

Démonstration. D'après les égalités (41), (82), (83), nous aurons

$$
\begin{aligned}
& +\int_{0}^{t} \iiint_{\Omega(Y)} \sum_{\beta=1}^{N} \hat{\Psi}_{X, t}^{(\alpha)}\left[W_{1 \beta}^{Y, \tau}(X, t ; Y, \tau), \ldots, W_{N \beta}^{Y, \tau}(X, t ; Y, \tau)\right] \varrho_{\beta}(Y, \tau) d Y d \tau \\
& +\int_{0}^{t} \iint_{E(I I)} \int_{\gamma=1} \sum_{\gamma}^{N} \hat{\Psi}_{X, t}^{(\alpha)}\left[W_{1 \gamma}^{I I, \zeta}(X, t ; \Pi, \zeta), \ldots, W_{N \gamma}^{i I, \zeta}(X, t ; \Pi, \zeta)\right] \bar{\varrho}_{\gamma}(\Pi, \zeta) d \Pi d \zeta .
\end{aligned}
$$

En substituant à la place de la fonction $\bar{\varrho}_{\alpha}$ resp. $\bar{\varrho}_{\gamma}$ l'expression (48) et en remarquant que les fonctions sous le signe des intégrales (48) et (88) admettent les singularités faibles $\left(72^{\prime \prime}\right)$, nous pouvons changer l'ordre d'intégration et nous aurons

$$
\begin{aligned}
&(89) \hat{\Psi}^{(\alpha)}\left[V_{1}(X, t) ; \ldots ; V_{N}(X, t)\right]=-\varrho_{\alpha}(X, t)- \\
&- \int_{0}^{t} \iiint_{\Omega(Y)} \sum_{\beta=1}^{N}\left\{\Phi_{\alpha \beta}(X, t ; Y, \tau)-\hat{\Psi}_{X, t}^{(\alpha)}\left[W_{1 \beta}^{Y, \tau}(X, t ; Y, \tau), \ldots, W_{N \beta}^{Y, \tau}(X, t ; Y, \tau)\right]\right. \\
&-\left.\int_{\tau}^{t} \iiint_{E(I)} \sum_{\nu=1}^{N} \hat{\Psi}_{X, t}^{(\alpha)}\left[W_{1 \gamma}^{\Pi, \zeta}(X, t ; \Pi, \zeta), \ldots, W_{N \gamma}^{\Pi, \zeta}(X, t ; \Pi, \zeta)\right] \Phi_{\gamma \beta}(\Pi, \zeta ; Y, \tau) d \Pi d \zeta\right\} \\
& \cdot \varrho_{\beta}(Y, \tau) d Y d \tau
\end{aligned}
$$

$(\alpha=1,2, \ldots, N)$. Or, les fonctions $\Phi_{\alpha \beta}$ forment les solutions du système d'équations intégrales (22), donc tout le terme intégral dans l'égalité (89) s'annule et nous arrivons au système d'égalités

$$
\hat{\Psi}^{(\alpha)}\left[V_{1}(X, t) ; \ldots ; V_{N}(X, t)\right]=-\varrho_{\alpha}(X, t)
$$

$(\alpha=1,2, \ldots, N)$, c.à $\mathrm{d}$. la thèse $(87) \mathrm{du}$ théorème 7 .

Les équations (87) sont analogues à l'équation de Poisson dans la théorie classique du potentiel.

ThÉonìme 8. Si les coefficients des équations (1) vérifient les conditions I, II, III, si en outre la densité continue $\left\{\varrho_{\beta}(Y, \tau)\right\}$ vérifie la condition de Hölder

$$
\left|\varrho_{\beta}(Y, \tau)-\varrho_{\beta}\left(Y_{1}, \tau\right)\right|<\varkappa_{\varrho}\left|Y Y_{1}\right|^{h_{\varrho}} \quad\left(0<h_{\varrho} \leqq 1\right)
$$

dans le domaine borné $\Omega$, pour $0 \leqq \tau \leqq T$, alors les dérivées spatiales d'ordre 
$M$ des composantes (39) du potentiel de charge spatiale vérifient la condition de Hölder de la forme

$$
\begin{aligned}
\mid D_{X}^{(M)}\left[V_{\alpha}(X, t)\right]-D_{X}^{(M)} & {\left[V_{\alpha}\left(X_{1}, t_{1}\right)\right] \mid } \\
& <\left(C_{1} M_{e}+C_{2} \varkappa_{e}\right)\left[\left|X X_{1}\right|^{h_{*}}+\left|t-t_{1}\right|^{\theta^{\prime} h /(h+M)}\right]
\end{aligned}
$$

dans tout domaine fermé $\Omega^{*}$ situé à l'intérieur du domaine $\Omega$ pour $t, t_{1} \in(0, T)$ où $h_{*}=h_{\varrho}$, si $h_{\rho}<h_{1}=\min \left(h, 2 h^{\prime}\right)$ et $h_{*}=\theta h_{1}$, si $h_{\varrho} \geqq h_{1} ; \theta, \theta^{\prime}$ sont des constantes positives arbitraires, inférieures à l'unité; $C_{1}$ et $C_{2}$ désignent les constantes positives dépendant du domaine $\Omega^{*}, M_{e}=\sup \left|\varrho_{\beta}\right|$.

Démonstration. D’après la décomposition (45), il suffit d'étudier les dérivées d'ordre $M$ du quasi-potentiel (46). Conformément aux trois sommes du membre droit de l'égalité (64), nous décomposons la dérivée d'ordre $M$ du quasi potentiel en trois sommandes

$$
D_{X}^{(M)}\left[V_{\alpha}^{*}(X, t)\right]=\Lambda_{1}(X, t)+\Lambda_{2}(X, t)+\Lambda_{3}(X, t) .
$$

Étudions d'abord l'intégrale

$$
\begin{array}{r}
\Lambda_{1}(X, t)=\int_{0}^{t} \sum_{\beta=1}^{N} \varrho_{\beta}(X, \tau)\left\{-\iint_{K^{\prime}} D_{X}{ }^{(M-1)}\left[W_{\alpha \beta}^{P, \tau}(X, t ; Q, \tau)\right] \cos v_{j} d \sigma_{Q}+\right. \\
\left.+\iiint_{\Omega-K} D_{X}{ }^{(M)}\left[W_{\alpha \beta}^{P, \tau}(X, t ; Y, \tau)\right] d Y\right\}_{P=X} d \tau
\end{array}
$$

On peut toujours admettre que le point $X$ dans le domaine $\Omega^{*}$ coïncide avec le centre $X_{0}$ de la sphere $K$ et que le rayon fixé $R_{K}$ de cette sphère est suffisamment petit pour que cette sphère soit situé dans le domaine $\Omega$. Il suffit d'étudier le cas où le second point arbitraire $X_{1}$ du domaine $\Omega$ se trouve à une distance $\left|X X_{1}\right|$ du centre $X_{0}$ de la sphère $K$ non-supérieure $\grave{a} \frac{1}{2} R_{K}$. Nous nous appuyons alors sur les inégalités (20), (59) et analogue pour les dérivées d'ordre $M$; nous nous appuierons, en outre, sur les inégalités

$$
\begin{aligned}
\mid D_{X}{ }^{(m)} & {\left[W_{\alpha \beta}^{Z, \zeta}(X, t ; Y, \tau)\right]-D_{X}^{(m)}\left[W_{\alpha \beta}^{Z_{1}, \zeta_{1}}(X, t ; Y, \tau)\right] \mid } \\
& <\frac{\text { const. }}{(t-\tau)^{\mu}} \cdot \frac{1}{|X Y|^{n+m-M \mu}} \sup \left|A_{\alpha j}^{k_{1} \ldots k_{n}}(Z, \zeta)-A_{\alpha j}^{k_{1} \ldots k_{n}}\left(Z_{1}, \zeta_{1}\right)\right|
\end{aligned}
$$

démontrées pour $m$ quelconque $(0<\mu<1$, si $m+n \geqq M$ et $0<\mu<$ $(m+n) M^{-1}$, si $\left.m+n<M\right)$ dans notre travail [5]. En décomposant d'une 
façon connue les différences des produits, figurant dans l'expression (93), en sommes des différences des facteurs, nous en concluons, sous l'hypothèse

$$
\left|X X_{1}\right| \leqq \frac{1}{2} R_{K}
$$

que la différence des valeurs de la fonction (93) aux points $X$ et $X_{1}$ vérifie l'inégalité de la forme

$$
\left|\Lambda_{1}(X, t)-\Lambda_{1}\left(X_{1}, t\right)\right|<\left(k_{1} M_{\varrho}+k_{2} \varkappa_{\varrho}\right)\left|X X_{1}\right|^{\alpha_{1}},
$$

où $\chi_{1}=\min \left(h_{e}, h\right), k_{1}$ et $k_{2}$ sont des constantes positives, dépendant du rayon fixé $R_{K}$ de la sphère $K$.

Étudions maintenant le terme intégrale

$$
\begin{aligned}
\Lambda_{2}(X, t)=\int_{0}^{t} \sum_{\beta=1}^{N} \varrho_{\beta}(X, \tau) \iiint_{\Omega}\{ & \left\{D_{X}{ }^{(M)}\left[W_{\alpha \beta}^{Y, \tau}(X, t ; Y, \tau)\right]-\right. \\
& \left.-\left(D_{X}{ }^{(M)}\left[W_{\alpha \dot{\beta}}^{P, \tau}(X, t ; Y, \tau)\right]\right)_{P=X}\right\} d Y d \tau
\end{aligned}
$$

indépendante de la sphère $K$. Considérons donc, comme antérieurement, une sphère $\omega$ de centre $X$, de rayon $R_{\omega}=2\left|X X_{1}\right| \leqq R_{K}$ et décomposons l'intégrale de volume (96) en deux intégrales

$$
\begin{aligned}
& \Lambda_{2}(X, t)=\Lambda_{2}^{\omega}(X, t)+\Lambda_{2}^{\Omega-\omega}(X, t), \\
& \Lambda_{2}\left(X_{1}, t\right)=\Lambda_{2}^{\omega}\left(X_{1}, t\right)+\Lambda_{2}^{\Omega-\omega}\left(X_{1}, t\right),
\end{aligned}
$$

étendues aux domaines $\omega$ et $\Omega-\omega$. D'après les inégalités (94), nous aurons

$$
\begin{aligned}
\left|\Lambda_{2}^{\omega}(X, t)\right| & <\int_{0}^{t} \frac{\text { const. } M_{\varrho} d \tau}{(t-\tau)^{\mu}} \int_{0}^{R_{\omega}} \frac{d r}{r^{M-M \mu-h+1}} \\
& =\text { const. } M_{\varrho} \cdot\left|X X_{1}\right|^{h-M(1-\mu)} \quad\left(1-h M^{-1}<\mu<1\right)
\end{aligned}
$$

et l'inégalité analogique pour le point $X_{1}$. Pour les intégrales étendues à la partie extérieure $\Omega-\omega$ on procède d'une façon analogue, que précédemment, en s'appuyant sur les inégalités (59), (94) et l'inégalité

On obtient

$$
|X Y| /\left|X_{1} Y\right| \leqq 2, \quad \text { si } \quad X_{1} \in \Omega-\omega .
$$

$$
\begin{aligned}
& \left|\Lambda_{2}^{\Omega-\omega}(X, t)-\Lambda_{2}^{\Omega-\omega}\left(X_{1}, t\right)\right| \\
& \quad<\text { const. }\left[\left|X X_{1}\right| \iiint_{\Omega-\omega} \frac{d Y}{|X Y|^{n+(M+1)(1-\mu)}}+\right. \\
& \left.\quad+\left|X X_{1}\right|^{h} \iiint_{\Omega-\omega} \frac{d Y}{|X Y|^{n+M-M \mu_{0}}}+\left|X X_{1}\right|^{h_{e}} \iiint_{\Omega-\omega} \frac{d Y}{|X Y|^{n+M-M \mu^{\prime} 0-h}}\right],
\end{aligned}
$$


où $\mu, \mu_{0}, \mu_{0}^{\prime}$ sont des constantes positives arbitraires, inférieures à l'unité. Il en résulte l'inégalité

$$
\left|\Lambda_{2}(X, t)-\Lambda_{2}\left(X_{1}, t\right)\right|<\left(k_{1}^{\prime} M_{e}+k_{2}^{\prime} \varkappa_{\varrho}\right)\left|X X_{1}\right|^{\chi_{2}},
$$

où $\chi_{2}=h_{\varrho}$, si $h_{e}<h$ et $\chi_{2}=\theta h$, si $h_{\varrho} \geqq h$.

Le troisième terme de la somme (92) a pour l'expression

(101) $\Lambda_{3}(X, t)=\int_{0}^{t} \iiint_{\Omega} \sum_{\beta=1}^{N} D_{X}{ }^{(M)}\left[W_{\alpha \beta}^{Y, \tau}(X, t ; Y, \tau)\right]\left[\varrho_{\beta}(Y, \tau)-\varrho_{\beta}(X, \tau)\right] d Y d \tau$.

Son étude est analogique au précédente et fournit l'inégalité de la forme (100). En réunissant les résultats (95) et (100), nous voyons que le quasi-potentiel vérifie l'inégalité de la forme

$$
\left|D_{X}^{(M)}\left[V_{\alpha}^{*}(X, t)\right]-D_{X}^{(M)}\left[V_{\alpha}^{*}\left(X_{1}, t\right)\right]\right|<\left(k_{1}^{\prime \prime} M_{\varrho}+k_{2}^{\prime \prime} \varkappa_{\varrho}\right)\left|X X_{1}\right|^{\chi_{2}} .
$$

Pour établir l'inégalité de Hölder de la fonction (92) par rapport à la variable $t$, remarquons d'abord que le centre $X$ de la sphère $K$ est extérieur au domaine d'intégration (93), donc la fonction (93) admet la dérivée par rapport à $t$ bornée dans $(0, T)$ et nous aurons la condition de Lipschitz

$$
\left|\Lambda_{1}(X, t)-\Lambda_{1}\left(X, t_{1}\right)\right|<\text { const. } M_{e} \cdot\left|t-t_{1}\right| .
$$

Pour étudier la fonction $\Lambda_{2}$ par rapport à $t$, écrivons $\left(t_{1}<t\right)$

(103) $\Lambda_{2}(X, t)-\Lambda_{2}\left(X, t_{1}\right)$

$$
\begin{gathered}
=\int_{t_{1}}^{t} \sum_{\beta=1}^{N} \varrho_{\beta}(X, \tau) \iiint_{\Omega}\left\{D_{X}{ }^{(M)}\left[W_{\alpha \beta}^{Y, \tau}(X, t ; Y, \tau)\right]-\left(D_{X}{ }^{(M)}\left[W_{\alpha \beta}^{P, \tau}(X, t ; Y, \tau)\right]\right)_{P=X}\right\} d Y d \tau \\
+\int_{0}^{t_{1}} \sum_{\beta=1}^{N} \varrho_{\beta}(X, \tau) \iiint_{\Delta}\left\{D_{X}{ }^{(M)}\left[W_{\alpha \beta}^{Y, \tau}(X, t ; Y, \tau)\right]-\left(D_{X}{ }^{(M)}\left[W_{\alpha \beta}^{P, \tau}(X, t ; Y, \tau)\right]\right)_{P=X}\right\} d Y d \tau \\
-\int_{0}^{t_{1}} \sum_{\beta=1}^{N} \varrho_{\beta}(X, \tau) \iiint_{\Delta}\left\{D_{X}{ }^{(M)}\left[W_{\alpha \beta}^{Y, \tau}\left(X, t_{1} ; Y, \tau\right)\right]-\left(D_{X}{ }^{(M)}\left[W_{\alpha \beta}^{P, \tau}\left(X, t_{1} ; Y, \tau\right)\right]\right)_{P=X}\right\} d Y d \tau \\
+\int_{0}^{t_{1}} \sum_{\beta=1}^{N} \varrho_{\beta}(X, \tau) \iiint_{\Omega}\left\{D_{X}{ }^{(M)}\left[W_{\alpha \beta}^{Y, \tau}(X, t ; Y, \tau)\right]-D_{X}{ }^{(M)}\left[W_{\alpha \beta}^{Y, \tau}\left(X, t_{1} ; Y, \tau\right)\right]\right. \\
\left.+\left(D_{X}{ }^{(M)}\left[W_{\alpha \beta}^{P, \tau}\left(X, t_{1} ; Y, \tau\right)\right]\right)_{P=X}-\left(D_{X}{ }^{(M)}\left[W_{\alpha \beta}^{P, \tau}(X, t ; Y, \tau)\right]\right)_{P=X}\right\} d Y d \tau,
\end{gathered}
$$

où $\Delta$ est une sphère de centre $X$ et de rayon $r_{\Delta}<R_{K}$ pas fixé pour le moment. Nous désignons les quatre intégrales du membre droit (103) 
successivement par $I_{1}, I_{2}, I_{3}, I_{4}$. D'après la limitation (94), la première des intégrales (103) vérifie l'inégalité

$$
\left|I_{1}\right|<\int_{t_{1}}^{t} \frac{\text { const. } M_{e} d \tau}{(t-\tau)^{\mu}} \cdot \iiint_{\Omega} \frac{d Y}{|X Y|^{n+M(1-\mu)-h}}<\text { const. } M_{e}\left|t-t_{1}\right|^{1-\mu}
$$

$\mu$ est une constante arbitraire à l'intérieur de l'intervalle $\left(1-h M^{-1}, 1\right)$.

Les intégrales $I_{2}$ et $I_{3}$ vérifient l'inégalité

$$
\left|I_{2,3}\right|<\text { const. } M_{e} \iiint_{\Delta} \frac{d Y}{|X Y|^{n+M(1-\mu)-h}}=\text { const. } M_{e} \cdot r_{\Delta}^{h-M(1-\mu)} .
$$

La limitation de la quatrième intégrale (103) sera basé sur la limitation de la dérivée (53) $(m=M)$, on obtient

(106) $\left|I_{4}\right|<$ const. $M_{\varrho} \cdot\left|t-t_{1}\right| \iiint_{\Omega-\Delta} \frac{d Y}{|X Y|^{n+M(2-\mu)}}<$ const. $M_{\varrho}\left|t-t_{1}\right| r_{\Delta}^{-M(2-\mu)}$.

Nous posons maintenant

$$
r_{\Delta}=\text { const. }\left|t-t_{1}\right|^{\alpha}<r_{K}
$$

et choisirons l'exposant positif $\alpha$ de façon qu'on ait

d'où résulte

$$
[h-M(1-\mu)] \alpha=1-M(2-\mu) \alpha,
$$

$$
\alpha=1 /(h+M) ;
$$

et il suit, d'après (104), (105), (106), la condition de Hölder suivante

$$
\left|\Lambda_{2}(X, t)-\Lambda_{2}\left(X, t_{1}\right)\right|<\text { const. } M_{\varrho}\left|t-t_{1}\right|^{\theta^{\prime} h /(h+M)},
$$

$\theta^{\prime}$ étant une constante arbitraire à l'intérieur de l'intervalle $(0,1)$.

L'étude de la troisième intégrale (101) est plus facile et évidente. En rapprochant les résultats (102) et (107), nous concluons que les dérivées d'ordre $M$ du quasi-potentiel vérifient l'inégalité

$$
\mid D_{X}{ }^{(M)}\left[V_{\alpha}^{*}(X, t)-D_{X}^{(M)}\left[V_{\alpha}^{*}\left(X, t_{1}\right)\right]\left|<k_{3} M_{\varrho}\right| t-\left.t_{1}\right|^{\theta^{\prime} h /(h+M)}\right.
$$

$k_{3}$ est une constante positive, dépendant du domaine $\Omega$.

Le second quasi-potential (47), d'après les propriétés (55') et (68) des fonctions $\bar{\varrho}_{\gamma}$, vérifie les mêmes propriétés $\left(101^{\prime}\right)$ et $\left(108^{\prime}\right)$, à la condition de substituer la constante $\theta h_{1}$ à la place de $h_{e}$. Il en résulte la thèse $(91) \mathrm{du}$ théorème 8 .

6. Application au problème de Cauchy. Les propriétés exprimées par les théorèmes 1 et 7 permettent de résoudre le problème de Cauchy pour le système parabolique d'équations de la forme 


$$
\hat{\Psi}^{(\alpha)}\left(u_{1}, u_{2}, \ldots, u_{N}\right)=F_{\alpha}(X, t)
$$

$(\alpha=1,2, \ldots, N)$ où les fonctions $F_{\alpha}(X, t)$ sont déterminées, bornées et continues dans la région

$$
X\left(x_{1}, \ldots x_{n}\right) \in E, \quad 0 \leqq t \leqq T
$$

et vérifient la condition de Hölder par rapport aux variables spatiales

$$
\left|F_{\alpha}(X, t)-F_{\alpha}\left(X_{1}, t\right)\right|<\text { const. }\left|X X_{1}\right|^{h_{F}}
$$

$\left(0<h_{F} \leqq 1\right)$. Les coefficients des opérations $\hat{\Psi}$ vérifient les conditions $I$, II, III, précisées dans l'introduction.

Conformément au problème de Cauchy, nous cherchons une suite de fonctions $u_{1}(X, t), \ldots, u_{N}(X, t)$ qui vérifient le système d'équations (109) en tout point $X$ de l'espace $E$ pour $0<t<T$ et qui satisfait à la condition initale

$$
\lim _{t \rightarrow 0} u_{\alpha}(X, t)=f_{\alpha}(X) \quad(x=1,2 \ldots, N),
$$

où les fonctions données $f_{\alpha}$ sont déterminées, bornées et continues dans tout l'espace $E$.

La solution du problème est donnée par les formules

$$
\begin{aligned}
u_{\alpha}(X, t)= & -\int_{0}^{t} \iiint_{E} \sum_{\beta=1}^{N} \Gamma_{\alpha \beta}(X, t ; Y, \tau) F_{\beta}(Y, \tau) d Y d \tau+ \\
& +\iiint_{E} \sum_{\beta=1}^{N} \Gamma_{\alpha \beta}(X, t ; Y, 0) f_{\beta}(Y) d Y
\end{aligned}
$$

$(\alpha=1,2, \ldots N)$, d'après les théorèmes 1 et 7 , qui peuvent être facilement généraliser pour tout l'espace $E$, en tenant compte des limitations $\left(28^{\prime}\right)$.

Il est facile, en tenant compte des mêmes limitations, de généraliser la résolution du problème aux cas où les fonctions données $F_{\alpha}$ et $f_{\alpha}$, en conservant la propriété (111) (si la distance $\left|X X_{1}\right|$ est inférieure à un nombre positif fixé) et la continuité, ne sont pas bornées dans l'espace $E$, mais vérifient les limitations

$$
\left\{\begin{array}{l}
\left|F_{\alpha}\right|<A\left|X X_{0}\right|^{p}+B, \\
\left|f_{\alpha}\right|<A\left|X X_{0}\right|^{p}+B ;
\end{array}\right.
$$

$A, B, p$ sont des constantes positives déterminées, $X_{0}$ - un point fixé de l'espace $E$. 


\section{TRAVAUX CITÉS}

1. S. D. Eidelman, Sur les solutions fondamentales des systèmes paraboliques, Mat. Sbornik N. S. 38 (80) (1956), 51-92. (En russe).

2. J. M. Gelfand et G. E. Silov, Transformation de Fourier des fonctions de croissance forte. Uspehi Matem. Nauk (N. S.) 8, no 6 (58) (1953), 3-54. (En russe.)

3. J. Petrovsky, Uber das Cauchysche Problem für ein System linearer partieller Differentialgleichungen im Gebiete der nichtanalytischen Funktionen, Bulletin de l'Université de Moscou 1 (1938), 65-67.

4. W. Pogorzelski, Étude de la solution fondamentale de l'équation parabolique, Ricerche Mat. 5 (1956), 25-57.

5. W. Pogorzelski, Étude de la matrice des solutions fondamentales du système parabolique. Ricerche Mat. 8 (1958), 153-185.

institut mathematique de LACAdémie polonaise des SCIENCES, Pologne 